Letter

\title{
Genetic combination risk for schizophrenia
}

Kengo Oishi $^{1 \S}$, Tomihisa Niitsu ${ }^{1}$, Nobuhisa Kanahara ${ }^{2}$, Tasuku Hashimoto ${ }^{1}$, Hideki Komatsu ${ }^{1}$, Tsuyoshi Sasaki ${ }^{1,3}$, Masayuki Takase $^{1}$, Yasunori Sato ${ }^{4}$, Masaomi Iyo ${ }^{1-3}$

${ }^{1}$ Department of Psychiatry, Chiba University Graduate School of Medicine, 1-8-1 Inohana, Chuou-ku, Chiba, Chiba 260-8670, Japan

${ }^{2}$ Division of Medical Treatment and Rehabilitation, Chiba University Center for Forensic Mental Health, 1-8-1 Inohana, Chuou-ku, Chiba, Chiba 260-8670, Japan

${ }^{3}$ Department of Child Psychiatry, Chiba University Hospital, 1-8-1 Inohana, Chuou-ku, Chiba, Chiba 260-8670, Japan

${ }^{4}$ Department of Preventive Medicine and Public Health, Keio University School of Medicine, 35 Shinanomachi Shinjuku-ku, Tokyo, 160-0016, Japan

${ }^{\S}$ Correspondence and requests for materials should be addressed to kengo.oishi@chiba-u.jp 


\section{Summary Paragraph}

2 Schizophrenia is a highly hereditary mental disease ${ }^{1}$ related to abnormal dopaminergic

3 activities. ${ }^{2,3}$ To elucidate the mechanisms underlying schizophrenia's development, genomic

4 studies have sought to identify the pathogenic genetic polymorphisms. Large-scale

5 genome-wide association studies (GWAS) have reported potential candidate loci that

6 contribute to schizophrenia's development. ${ }^{4,5}$ The risk genetic profiles are not yet established.

7 Here we show that the combination of three functional single nucleotide polymorphisms

8 (SNPs) related to the key factors of dopaminergic signaling can be used to predict the risk of

9 schizophrenia's development, though none of the SNPs is known to be associated by itself.

10 These functional SNPs were reported to demonstrate directional influences in their parent

11 gene activity, perhaps characterizing the integrated properties of dopaminergic signaling.

12 Interestingly, the risk combination presented here included the major genotype as well as the

13 minor polymorphisms, suggesting a possible association of unaffected activities of some

14 dopamine-related genes with the disease development. The phenotype speculated based on

15 the allelic status seemed consistent with the conventional pathophysiological hypotheses,

16 although recently developed predictive methods, such as the polygenic risk score, could miss

17 this potent pathogenic role of carrying a normal genotype by evaluating only minor

18 polymorphisms. Our results demonstrate the presence of a subtype in schizophrenia with the

19 favored genetic background related to dopamine signaling. Our findings indicate the

20 possibility that the combinations could characterize integrated biological functions (including

21 neurotransmission) and therefore identify individuals with a disease risk. The biological

22 microenvironment indicated by the functional SNPs could bring an insight to elucidate the

23 pathogenic mechanisms of developing schizophrenia. Furthermore, we believe that our 
1 approach will contribute to the development of innovative means to predict disease risks even

2 for other multi-factorial diseases and then, the following preventive medicine. 


\section{Main}

2 Schizophrenia is a disabling chronic mental illness, consisting of the prodromal phase and

3 following psychotic onset, with the lifetime prevalence of $0.4 \%$ to $1.0 \%$ worldwide. ${ }^{6,7}$ The

4 majority of first-episode schizophrenia patients positively respond to antipsychotic treatment

5 with dopamine D2 receptor (DRD2) antagonists. ${ }^{2}$ Increased dopamine synthesis was

6 observed in these responding patients, ${ }^{3}$ suggesting the involvement of abnormal

7 dopaminergic signaling in the pathology of schizophrenia. Although epidemiologic studies

8 convincingly suggested the high genetic heritability of schizophrenia, ${ }^{1}$ genome-wide

9 association studies (GWAS) have not succeeded in indicating the apparent association of

10 dopamine-related genes with schizophrenia. ${ }^{8}$ In fact, those studies tended to obtain negative

11 results regarding the genetic associations with schizophrenia among the candidates proposed

12 by a variety of psycho-pharmacological hypotheses. ${ }^{9}$

We recently demonstrated that analyses of the combinations of multiple functional

14 single nucleotide polymorphisms (SNPs) of the parent genes that are key factors in

15 dopaminergic signaling could be used to predict the risk of developing late-onset

16 treatment-resistant schizophrenia (L-TRS). ${ }^{10}$ L-TRS may be due to antipsychotic-induced

17 dopamine supersensitivity; ${ }^{11,12}$ i.e., L-TRS may be caused by the compensatory up-regulation

18 of DRD2. ${ }^{13-15}$ Because individuals with L-TRS may be correctly classified as having typical

19 dopamine-related schizophrenia, we evaluated the combination of dopamine-related

20 functional SNPs, i.e., rs10770141 of the tyrosine hydroxylase $(T H)$ gene, rs4680 of the

21 catechol-O-methyltransferase (COMT) gene, and rs1800497, also called TaqIA, of the DRD2

22 gene (Table 1). We identified two potent risk combinations for L-TRS; one is the double SNP

23 combination of $\mathrm{T}(+)$ of $\mathrm{rs} 10770141$ and $\operatorname{Met}(-)$ of rs 4680 , and the other is the triple SNP 
1 combination of $\mathrm{T}(+)$ of rs 10770141, Met(-) of rs4680, and A1(+) of rs1800497. It has been

2 reported that these individual SNPs may reflect the relatively higher $T H$ gene expression ${ }^{16}$

3 and COMT activity. ${ }^{17}$ In addition, the individuals with $\mathrm{A} 1(+)$ of rs 1800497 could have lower

$4 \quad D R D 2$ gene expression. ${ }^{18}$ Since these dopaminergic characteristics could be associated with

5 schizophrenia, we tested here whether these allelic combinations could pose a risk for the

6 development of the disease.

$7 \quad$ Our case-control study included 361 schizophrenic patients and 282 healthy controls

8 (Suppl. Table S1). None of the included SNPs showed an allelic or genotypic association with

9 schizophrenia (Suppl. Table S2). However, we found that the individuals with the triple

10 combination were significantly associated with schizophrenia (odds ratio [OR] 5.56, 95\%

11 confidence interval [CI] 1.25-24.69, $\mathrm{p}=0.011$ ), whereas the double combination showed a

12 tendency to be associated with schizophrenia (OR 2.54, 95\%CI 1.00-6.46, p=0.062) (Tables

$132,3)$. Within the total of 16 individuals who carried the triple combination, 14 had been

14 diagnosed as having schizophrenia.

Although we observed that none of the single SNPs was associated with schizophrenia,

16 a finding that is consistent with most of the literature, the significant association of the unique

17 triple combination might indicate that individuals with schizophrenia have some inborn

18 dopaminergic properties. Individuals with $\mathrm{T}(+)$ and $\operatorname{Met}(-)$ might be expected to show

19 relatively higher dopamine synthesis and more rapid dopamine degradation, which also

20 suggests that there are more dynamic alterations in dopamine concentrations at the synaptic

21 cleft. In addition, carrying A1(+) might be associated with a lower presynaptic DRD2 density

22 and consequently an enhanced dopamine release due to the attenuated negative feedback

23 loops. ${ }^{19}$ Thus, these individuals might exhibit relatively more pulsatile dopamine signaling. 
It is known that repeated exposure to dopamine agonists (such as psychostimulants ${ }^{20}$ )

2 and/or stressors ${ }^{21}$ causes sensitization. The term 'sensitization' means a progressive and

3 long-lasting amplification of the behavioral and neurochemical response $\mathrm{e}^{22}$ and has been

4 argued to play a pathogenic role in causing not only psychosis but also schizophrenia. ${ }^{23-25}$ In

5 fact, it is clinically suggested that frequent exposure to stress poses a risk for prodromal

6 symptoms and schizophrenia. ${ }^{26,27}$ Individuals with schizophrenia were reported to release

7 greater amounts of dopamine in response to stress. ${ }^{6}$ Individuals who carry the risk genetic

8 combination might demonstrate similar dopaminergic pathogenesis, since relatively rapid

9 pulsatile signaling could be caused repeatedly in response to daily stressors. This similarity in

10 dopaminergic pathogenesis could contribute to sensitization and be a reason for the increased

11 likelihood of schizophrenia onset. It may also be well consistent with the observations that

12 both genetic and environment factors seem to present risks for schizophrenia. ${ }^{28,29}$

Our pathological hypothesis of schizophrenia demonstrated in this report accurately

14 accounts for the recently proposed clinical picture of schizophrenia. ${ }^{6,25}$ Assuming that the

15 sensitization to internal dopamine is somehow involved in the pathogenic mechanism of

16 schizophrenia, the identification of the at-risk individuals at a preclinical stage might provide

17 psychiatrists a pivotal chance to prevent the onset by managing the individual's pulsatile

18 dopamine activities. The risk genotypic combination discovered in the present study were

19 detected in only $4.0 \%$ of the schizophrenic patients. However, the occurrence rate of the

20 disease within the risk population was $87.5 \%$, where the 14 out of 16 individuals were

21 diagnosed to schizophrenia. Furthermore, although the polygenic risk score was reported to

22 show no association with TRS $^{31}$, the 9 out of these 14 schizophrenic patients $(64.3 \%)$ were

23 classified as TRS (Suppl. Table S3). We speculate that the inclusion rate could be improved 
1 by evaluating better combinations of other functional SNPs related to the dopamine pathway. In addition, although the lowest MAF (5.7\%, of rs10770141) was the apparently

3 narrowing polymorphism in our study population, the Trans-Omics for Precision Medicine

4 project recently reported to the U.S. National Center for Biotechnology Information that the

$5 \quad$ MAF was estimated to be as large as $42.9 \%$ in the global population, suggesting its

6 applicability. ${ }^{30}$ In the present study, we focused on the genetic variants related to the

7 dopaminergic system, but our approach will be appropriate to assess other candidates related

8 to different neurotransmissions including gamma-aminobutyric acid (GABA) and

$9 \quad$ glutamatergic signaling.

10 There are some issues to be considered as study limitations. First, the mean age of our

11 healthy control subjects was significantly lower than that of the patients. Although

12 schizophrenia generally emerges during adolescence, our controls could still include potent

13 false negatives. However, the impact may be limited because (1) the mean age of disease

14 onset was significantly younger than the mean age of the controls, and (2) the lifetime

15 prevalence of schizophrenia was estimated to be as small as $0.4 \%-1.0 \%$. In addition, our

16 sample size was large enough to allow us to evaluate our results, but further validations are

17 clearly expected $\square-\square$ including those obtained for large and multi-ethnic populations.

18 In conclusion, we conducted a case-control study to investigate the association of

19 functional SNPs related to dopamine transmission with schizophrenia. Our analyses revealed

20 that the combination of the SNPs related to $T H, C O M T$ and $D R D 2$ genes was significantly

21 associated with the onset of schizophrenia. We hypothesize that the present genetic

22 combination characterizes dopamine transmission. The findings are important because of

23 their potential to bring new insights to risk prediction and the development of new treatment 
bioRxiv preprint doi: https://doi.org/10.1101/478958; this version posted July 18,2019 . The copyright holder for this preprint (which was not certified by peer review) is the author/funder. All rights reserved. No reuse allowed without permission.

1 methods for schizophrenia. However, future studies are necessary to test our findings.

2 


\section{References}

1. Hilker R et al. Heritability of schizophrenia and schizophrenia spectrum based on the nationwide Danish Twin Register. Biol Psychiatry. 2018; 83: 492-498.

2. Agid $\mathrm{O}$ et al. An algorithm-based approach to first-episode schizophrenia: Response rates over 3 prospective antipsychotic trials with a retrospective data analysis. J Clin Psychiatry. 2011; 72: 1439-1444.

3. Demjaha A, Murray RM, McGuire PK, Kapur S, Howes OD. Dopamine synthesis capacity in patients with treatment-resistant schizophrenia. Am J Psychiatry. 2012; 169: 1203-1210.

4. Schizophrenia Working Group of the Psychiatric Genomics C. Biological insights from 108 schizophrenia-associated genetic loci. Nature. 2014; 511: 421-427.

5. Zhang JP et al. Schizophrenia polygenic risk score as a predictor of antipsychotic efficacy in first-episode psychosis. Am J Psychiatry. 2018 Nov 5. doi:

10.1176/appi.ajp.2018.17121363.

6. Howes OD, McCutcheon R, Owen MJ and Murray R. The role of genes, stress and dopamine in the development of schizophrenia. Biol Psychiatry. 2017; 81: 9-20.

7. Saha S et al. A systematic review of the prevalence of schizophrenia. PLoS Med. 2005; 2: e141.

8. Edwards AC, Bacanu S, Bigdeli TB, Moscati A, Kendler KS. Evaluating the dopamine hypothesis of schizophrenia in a large-scale genome-wide association study. Schizophr Res. 2016; 176: 136-140.

9. Johnson EC et al. No evidence that schizophrenia candidate genes are more associated with schizophrenia than noncandidate genes. Biol Psychiatry. 2017; 82: 702-708.

10. Oishi K et al. Vulnerable combinations of functional dopaminergic polymorphisms to late-onset treatment resistant schizophrenia. PLOS ONE. 2018; 13: 0207133.

11. Silvestri S et al. Increased dopamine D2 receptor binding after long-term treatment with antipsychotics in humans: Clinical PET study. Psychopharmacology (Berl). 2000; 152: 174-180.

12. Seeman MV, Seeman P. Is schizophrenia a dopamine supersensitivity psychotic reaction? Prog Neuropsychopharmacol Biol Psychiatry. 2014; 48: 155-160.

13. Chouinard G, Jones BD, Annable L. "Neuroleptic-induced supersensitivity psychosis". Am J Psychiatry. 1978; 135: 1409-1410. 
14. Chouinard $\mathrm{G}$ et al. Antipsychotic-induced dopamine supersensitivity psychosis: Pharmacology, criteria, and therapy. Psychother Psychosom. 2017; 86: 189-219.

15. Iyo $\mathrm{M}$ et al. Optimal extent of dopamine D2 receptor occupancy by antipsychotics for treatment of dopamine supersensitivity psychosis and late-onset psychosis. J Clin Psychopharmacol. 2013; 33: 398-404.

16. Horiguchi $\mathrm{M}$ et al. Functional polymorphism (C-824T) of the tyrosine hydroxylase gene affects IQ in schizophrenia. Psychiatry Clin Neurosci. 2014; 68: 456-462.

17. Weinshilboum RM, Otterness DM, Szumlanski C. Methylation pharmacogenetics: Catechol O-methyltransferase, thiopurine methyltransferase, and histamine N-methyltransferase. Annu Rev Pharmacol Toxicol. 1999; 39: 19-52.

18. Arinami T, Gao M, Hamaguchi H, Toru M. A functional polymorphism in the promoter region of the dopamine D2 receptor gene is associated with schizophrenia. Hum Mol Genet. 1997; 6: 577-582.

19. Ford CP. The role of D2-autoreceptors in regulating dopamine neuron activity and transmission. Neuroscience. 2014; 282: 13-22.

20. Ujike H, Sato M. Clinical features of sensitization to methamphetamine observed in patients with methamphetamine dependence and psychosis. Ann NY Acad Sci. 2004; 1025: 279-287. Review.

21. Kalivas PW1, Stewart J. Dopamine transmission in the initiation and expression of drugand stress-induced sensitization of motor activity. Brain Res Brain Res Rev. 1991; 16: 223-244.

22. Nestler EJ. Molecular mechanisms of drug addiction. Neuropharmacology. 2004; 47: 24-32.

23. Sato M, Chen CC, Akiyama K, Otsuki S. Acute exacerbation of paranoid psychotic state after long-term abstinence in patients with previous methamphetamine psychosis. Biol Psychiatry. 1983; 18: 429-440.

24. Sato M, Numachi Y, Hamamura T. Relapse of paranoid psychotic state in methamphetamine model of schizophrenia. Schizophr Bull. 1992; 18: 115-122. Review.

25. Weidenauer A et al. Making sense of: Sensitization in schizophrenia. Int $J$ Neuropsychopharmacol. 2016; 20: 1-10.

26. Day R et al. Stressful life events preceding the acute onset of schizophrenia: A cross-national study from the World Health Organization. Cult Med Psychiatry. 1987; 11: 
123-205.

27. Tessner KD, Mittal V, Walker EF. Longitudinal study of stressful life events and daily stressors among adolescents at high risk for psychotic disorders. Schizophr Bull. 2011; 37: 432-441.

28. Bayer TA, Falkai P, Maier W. Genetic and non-genetic vulnerability factors in schizophrenia: The basis of the "two hit hypothesis." J Psychiatr Res. 1999; 33: 543-548.

29. van Os J, Kenis G, Rutten BP. The environment and schizophrenia. Nature. 2010; 468: 203-212.

30. The U.S. National Center for Biotechnology Information: The database of Short Genetic Variations, dbSNP. https://www.ncbi.nlm.nih.gov/snp/rs10770141\#frequency_tab.

Accessed Nov. 19, 2018.

31. Wimberley T et al. Polygenic Risk Score for Schizophrenia and Treatment-Resistant Schizophrenia. Schizophr Bull. 2017 Sep 1;43(5):1064-1069. 
Table 1. The functional SNPS

\begin{tabular}{|l|c|c|c|c|c|}
\hline \multicolumn{1}{|c|}{ SNP ID } & $\begin{array}{c}\text { Related } \\
\text { gene }\end{array}$ & \multicolumn{1}{|c|}{ Polymorphism function [ref.] } & WT & $\begin{array}{c}\text { Poly- } \\
\text { morphism }\end{array}$ & MAF \\
\hline rs10770141 & TH & $30 \%-40 \%$ higher gene expression [16] & C & T & $6.4 \%$ \\
rs4680 & COMT & $50 \%-75 \%$ reduction of the enzymatic activity [17] & Valine & Methionine & $33.8 \%$ \\
rs1800497 & DRD2 & $12 \%$ less availability of striatal DRD2 [18] & A2 & A1 & $35.3 \%$ \\
\hline
\end{tabular}

${ }^{\mathrm{a}} \mathrm{MAFs}$ (minor allele frequencies) were estimated in all of the study subjects (Suppl. Table S1). 
Table 2. The double combination of dopamine-related functional SNPs

\begin{tabular}{|l|cc|rc|rl|}
\hline \multirow{2}{*}{$\begin{array}{c}\text { Double } \\
\text { combination }\end{array}$} & \multicolumn{2}{|c|}{$\begin{array}{c}\text { Schizophrenia } \\
\text { patients }\end{array}$} & \multicolumn{2}{c|}{$\begin{array}{c}\text { Healthy } \\
\text { controls }\end{array}$} & \multicolumn{2}{|c|}{ Total } \\
\cline { 2 - 8 } & $\mathrm{n}$ & Proportion & $\mathrm{n}$ & Proportion & $\mathrm{n}$ & Proportion \\
\hline Candidate $^{\mathrm{a}}$ & 19 & $5.4 \%$ & 6 & $2.2 \%$ & 25 & $4.0 \%$ \\
The rest & 336 & $94.6 \%$ & 270 & $97.8 \%$ & 606 & $96.0 \%$ \\
\hline Total & 355 & $100.0 \%$ & 276 & $100.0 \%$ & 631 & $100.0 \%$ \\
\hline
\end{tabular}

The association of the candidate combination was evaluated by Fisher's exact test.

${ }^{\mathrm{a}}$ The candidate double combination was $\mathrm{T}(+)$ of rs 10770141 and Met(-) of rs4680, which showed a tendency to be related to schizophrenia (OR 2.54, $95 \% \mathrm{Cl}$

$1.00-6.46, p=0.062)$. The missing data: Six (1.7\%) schizophrenia patients, six $(2.1 \%)$ healthy controls; thus $12(1.9 \%)$ for the total. 
Table 3. The triple combination of dopamine-related functional SNPs

\begin{tabular}{|c|c|c|c|c|c|c|}
\hline \multirow{2}{*}{$\begin{array}{l}\text { Triple } \\
\text { combination }\end{array}$} & \multicolumn{2}{|c|}{ Schizophrenia } & \multicolumn{2}{|c|}{ Healthy controls } & \multicolumn{2}{|r|}{ Total } \\
\hline & $n$ & Proportion & $\mathrm{n}$ & Proportion & $\mathrm{n}$ & Proportion \\
\hline Candidate $^{a}$ & 14 & $4.0 \%$ & 2 & $0.7 \%$ & 16 & $2.5 \%$ \\
\hline The rest & 341 & $96.0 \%$ & 271 & $99.3 \%$ & 612 & $97.5 \%$ \\
\hline Total & 355 & $100.0 \%$ & 273 & $100.0 \%$ & 628 & $100.0 \%$ \\
\hline
\end{tabular}

The association of the candidate combination was evaluated by Fisher's exact test.

${ }^{a}$ The candidate triple combination was $\mathrm{T}(+)$ of rs 10770141 , Met(-) of rs4680, and $\mathrm{A} 1(+)$ of $\mathrm{rs} 1800497$, which showed a significant association with schizophrenia (OR $5.56,95 \% \mathrm{Cl} 1.25-24.69, \mathrm{p}=0.011)$.

The missing data: Six (1.7\%) patients in the schizophrenia group, nine $(3.2 \%)$ healthy controls; thus $15(2.8 \%)$ for the total. 
All the authors have no conflicts of interests to disclose and approved this submission.

Correspondence and requests for materials should be addressed to kengo.oishi@chiba-u.jp. 